\title{
Deterioro cognitivo en poblaciones urbanas y rurales de la mediana altura geográfica en Bolivia: Prevalencia y factores asociados.
}

\section{Cognitive Impairment in Urban and Rural Populations of the Middle Altitude in Bolivia: Prevalence and Associated}

Factors

Christian R. Mejia1a, Cecilia Fernandez-Ledezma2a, Eddy Cossio-Andia3b.

\begin{abstract}
Introduction: Cognitive impairment is an important pathology among the Aged, but few studies evaluate populations at an important geographical height. Objectives: To characterize and find the factors associated with cognitive deterioration in urban and rural populations of medium geographic height. Methodology: Analytical crosssectional study, cognitive impairment was evaluated with the Pfeiffer Test (Cronbach's Alpha: 0.69), its results were combined versus three socio-educational variables and four comorbidities; analytical statistics were obtained. Results: Of the 400 older adults, 26.5\% (106) had no cognitive impairment, 47.5\% (190) had mild impairment, $25.5 \%$ (102) moderate and $0.5 \%$ (2) severe; $60 \%$ were women, the median age was 66 years (interquartile range: 63-69 years), 67\% lived in rural areas. In the multivariate analysis it was found that there was more moderate / severe cognitive impairment at older age (RPa: 1.05; 95\% CI: 1.02-1.08; $p<0.001$ ), among those with diabetes (RPa: 1 , 23; 95\% CI: 1.17-1.29; $p<0.001$ ), arterial hypertension (RPa: 1.47; 95\% CI: 1.331.61; $p<0.001$ ), chronic kidney disease (RPa: 2.21, 95\% CI 2.01-2.43, $p<0.001$ ) and heart failure (RPa: 2.25, 95\% CI 1.92-2.64, $p<0.001$ ); On the other hand, those with a university degree had less cognitive deterioration (RPa: 0.89, 95\% CI: 0.22-0.65, $p$ $<0.001)$; adjusted for sex and place of residence. Conclusion: Important associations of cognitive deterioration were found in an older adult population residing between 2500-3900 msnm, these results are important as they are not a highly studied population; therefore, these data should be taken for diagnosis and treatment.
\end{abstract}

Keywords: Cognitive Dysfunction, Altitude, Aged.

Rev Chil Neuro-Psiquiat 2021; 59 (4): 280-288

Los autores declaran no tener conflictos de intereses.

Aceptado: 2021/03/04

Recibido: 2021/01/30

Universidad Continental. Lima, Perú.

Universidad Mayor de San Simón. Cochabamba, Bolivia.

Incubadora de Investigación en Salud. Cochabamba, Bolivia.

Doctor en Medicina.

Estudiante de Medicina 


\section{INTRODUCCIÓN}

$E_{\text {la }}^{1}$ 1 envejecimiento es parte del proceso de la evolución. En los últimos años, gracias a la mejora de la calidad de vida se ha observado un aumento de la población adulta mayor en muchos lugares del mundo(1), lo que ha inducido a un incremento de la prevalencia de enfermedades crónicas y, por lo tanto, una mayor necesidad de atención hospitalaria ${ }^{(2,3)}$.

Por otro lado, el aumento de la población senil se ha reportado en múltiples regiones, inclusive en aquellas consideradas inhóspitas, como las ciudades a gran altura. Esto sumado a los efectos propios del ambiente puede repercutir directamente sobre la salud de estos individuos. Es así que, un estudio realizado en la India (a más de 4300 metros sobre el nivel del mar) mostró el aumento de la prevalencia del deterioro cognitivo leve en la población que permanecen por más tiempo a gran altitud, presumiblemente por la hipoxia global a gran altitud, que podría afectar negativamente en el rendimiento $\operatorname{cognitivo}^{(4)}$.

Es importante comprender los mecanismos subyacentes y diferencias en el envejecimiento cognitivo, algunos pueden tener una capacidad cognitiva conservada, mientras que otros presentan una disminución, que es perjudicial para su bienestar, independencia y, principalmente, su calidad de vida ${ }^{(5,6)}$. De manera que, las principales alteraciones observadas durante este proceso de pérdida cognitiva se caracterizan por enlentecimiento, déficit inhibitorio, déficit de transmisión, declinación de la memoria operativa y procesamiento del autorregulado del lenguaje $\mathrm{e}^{(7)}$. A su vez, la aparición de este deterioro se asocia a daño cerebral, que a medida que se vuelve más evidente puede traducirse en un riesgo incrementado de sufrir Enfermedad de Alzheimer u otras enfermedades neurodegenerativas ${ }^{(8)}$. Siendo esto importante en grandes ciudades, como el caso de Cochabamba, donde casi un tercio de millón de habitantes tiene más de 60 años $^{(9)}$. Es por esto que, el objetivo fue el caracterizar y encontrar los factores asociados al deterioro cognitivo en poblaciones urbanas y rurales de la mediana altura geográfica.

\section{METODOLOGÍA}

\section{Diseño y población}

Se realizó un estudio de tipo transversal analítico, que se basó en una data secundaria, ya que, la información se tomó de un registro que realizó una de las autoras como parte de su informe anual para la residencia de geriatría.

Se incluyó a los pacientes adultos mayores que se atendieron en el hospital Clinico Viedma, que se encontraba a 2587 metros sobre el nivel del mar, que tuviesen una evaluación de su deterioro cognitivo y que tuviesen las variables completas. No se excluyó pacientes, debido a que se contaban con todas las fichas de evaluación completas.

El muestreo fue de tipo no aleatorio, para saber si es que se tuvo un buen tamaño muestral se realizó un cálculo de la potencia de cada uno de los cruces del tener deterioro cognitivo moderado/severo versus las otras variables: sexo (33\%), nivel educativo $(100 \%)$, diabetes mellitus (14\%), hipertensión arterial $(70 \%)$, enfermedad renal crónica $(100 \%)$, insuficiencia cardiaca $(100 \%)$ y lugar de residencia (57\%).

\section{Variables y procedimientos}

La variable dependiente fue el deterioro cognitivo, que fue evaluada mediante el Test de Pfeiffer, que en la medición del Alpha de Cronbach se obtuvo un valor de 0,69. Este test consta de 10 preguntas, que evalúan la memoria a corto y largo plazo, orientación, información sobre hechos cotidianos y capacidad para realizar un trabajo matemático seriado $^{(10)}$. En otras investigaciones se ha mostrado que este test tiene una sensibilidad 
del $91 \%$ y una especificidad del 90\%, además, es una prueba de fácil administración, no requiere ningún material específico para su realización y se puede aplicar a los adultos mayores con bajo nivel educativo ${ }^{(11,12)}$. Se realiza en un tiempo aproximado de 5-10 minutos, y con un resultado de 3 o más errores (4 o más en caso de analfabetismo) es sugestivo de deterioro cognitivo ${ }^{(13)}$.

Las otras variables fueron el sexo (masculino o femenino), la edad (tomada en su forma cuantitativa), el nivel académico (categorizado como universitarios o no universitarios, este último tuvo a los niveles: primario, secundario y técnico), el tener alguna de las comorbilidades evaluadas (que fueron recolectadas por ser las variables más importantes para el tema de deterioro cognitivo ${ }^{(3)} \mathrm{y}$ el lugar de residencia (urbano o rural).

Para la obtención de datos se utilizó una planilla para el registro de las respuestas que posteriormente se tabularon al programa Excel, estos fueron recogidos en consulta externa del Hospital Clinico Viedma.

\section{Análisis de datos y ética}

El análisis de datos se realizó en el programa estadístico Stata 11,1 (licencia adquirida por uno de los autores). Primero se realizó la estadística descriptiva, en donde se obtuvo las frecuencias y porcentajes de las variables categóricas y las medianas y rangos intercuartílicos de las variables cuantitativas (por tener un comportamiento no normal); en esta etapa se obtuvo los valores $\mathrm{p}$, con la prueba de chi cuadrado y la suma de rangos (para el cruce versus variables categóricas y cuantitativas; respectivamente). Luego se obtuvo las razones de prevalencia, los intervalos de confianza al 95\% (IC95\%) y valores p; todos mediante los modelos lineales generalizados (con la familia Poisson, la función de enlace log, modelos robustos y ajustados por el lugar de residencia). Se consideró como estadísticamente significativos a los valores $\mathrm{p}<0,05$.
En cuanto a la ética se pidió a los participantes el consentimiento informado, se protegió los datos de los participantes para que no puedan ser identificados.

\section{RESULTADOS}

De los 400 adultos mayores, el 59,8\% (239) fueron mujeres, la mediana de edades fue de 66 años (rango intercuartílico: 63-69 años), el 66,5\% (266) vivían en la zona rural, el 32,0\% (128) tenían diabetes mellitus, el 34,8\% (139) hipertensión arterial, el 5,0\% (20) enfermedad renal crónica y el $26,8 \%$ (107) insuficiencia cardiaca. Según el lugar de residencia, no hubo diferencias estadísticamente significativas según el sexo $(p=0,054)$, la edad $(p=0,297)$, el tener diabetes mellitus $(\mathrm{p}=0,513)$, hipertensión arterial $(p=0,152)$, la enfermedad renal crónica $(\mathrm{p}=0,264)$ o la insuficiencia cardiaca $(p=0,450)$; si hubo diferencia en del nivel educativo $(\mathrm{p}<0,001)$. Tabla 1

E1 26,5\% (106) no tuvo deterioro cognitivo, el 47,5\% (190) tuvo un deterioro leve, el $25,5 \%$ (102) moderado y el $0,5 \%$ (2) severo. Cuando se realizó el cruce según el lugar de residencia no hubo asociación estadística $(p=0,503$ con la prueba del chi cuadrado), los que vivían en la zona rural tuvieron $68 \%$, $69 \%, 61 \%$ y $50 \%$ de parámetros normales, deterioro cognitivo leve, moderado y severo; respectivamente. Figura 1

(Cuando se realizó el análisis del deterioro cognitivo, se encontró que hubo diferencias estadísticas según la edad (los que tuvieron deterioro cognitivo moderado $\mathrm{o}$ severo tuvieron mayor edad; $p<0,001$ ), según el tener enfermedad renal crónica (los enfermos tuvieron casi el doble de deterioro cognitivo; $p=0,047$ ) y el tener insuficiencia cardiaca (los enfermos tuvieron más del doble de deterioro cognitivo; $p<0,001)$. Tabla 2

En el análisis multivariado se encontró que, hubo más deterioro cognitivo moderado/severo a mayor edad (RPa: 1,05; IC95\%: 
Tabla 1. Características de la población según el lugar de residencia de los adultos mayores de Cochabamba-Bolivia.

\begin{tabular}{lccc}
\hline \multirow{2}{*}{ Variables } & \multicolumn{2}{c}{ Reside en zona } & \multirow{2}{*}{ Valor p } \\
\cline { 2 - 3 } Sexo & Urbano (n=134) & Rural (n=266) & \\
$\quad$ Masculino & $45(28,0 \%)$ & $116(72,0 \%)$ & 0,054 \\
Femenino & $89(37,2 \%)$ & $150(62,8 \%)$ & \\
Edad (años)* & $65(62-69)$ & $66(63-69)$ & 0,297 \\
Nivel educativo & & & \\
$\quad$ Hasta técnico & $119(31,3 \%)$ & $261(68,7 \%)$ & $<0,001$ \\
Universitario & $15(75,0 \%)$ & $5(25,0 \%)$ & \\
Diabetes Mellitus & $94(34,6 \%)$ & $178(65,4 \%)$ & \\
$\quad$ No & $40(31,3 \%)$ & $88(68,7 \%)$ & \\
Si & & & \\
Hipertensión arterial & $81(31,0 \%)$ & $180(69,0 \%)$ & 0,152 \\
No & $53(38,1 \%)$ & $86(61,9 \%)$ & \\
Si & & & \\
Enfermedad renal crónica & $125(32,9 \%)$ & $255(67,1 \%)$ & 0,264 \\
No & $9(45,0 \%)$ & $11(55,0 \%)$ & \\
Si & & & \\
Insuficiencia cardiaca & $95(32,4 \%)$ & $198(67,6 \%)$ & 0,45 \\
No & $39(36,5 \%)$ & $68(63,5 \%)$ & \\
Si & & & \\
\hline
\end{tabular}

Los valores p se obtuvieron con la prueba de chi cuadrado y la suma de rangos (para la edad). * La edad fue tomada de forma cuantitativa y los valores que se muestran son las medianas (rangos intercuartílicos).

Figura 1. Porcentajes del deterioro cognitivo según el residir en zona rural o urbana de los adultos mayores de Cochabamba-Bolivia.

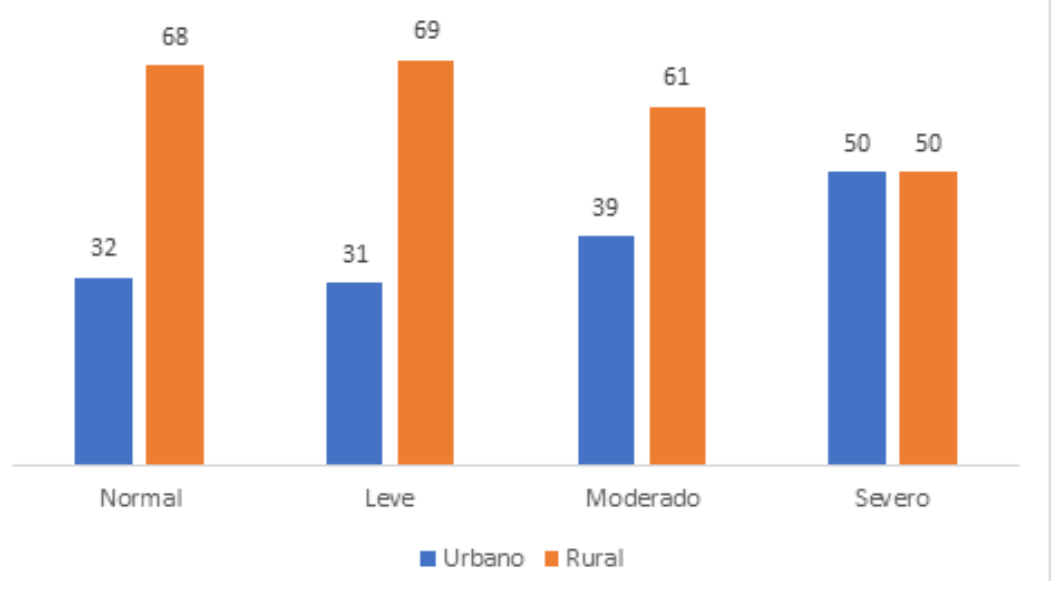


Tabla 2. Características de la población según el lugar de residencia de los adultos mayores de Cochabamba-Bolivia.

\begin{tabular}{|c|c|c|c|}
\hline \multirow{2}{*}{ Variables } & \multicolumn{2}{|c|}{ Deterioro cognitivo } & \multirow{2}{*}{ Valor $\mathbf{p}$} \\
\hline & No o leve & Moderado o severo & \\
\hline \multicolumn{4}{|l|}{ Sexo } \\
\hline Masculino & $114(70,8 \%)$ & $47(29,2 \%)$ & 0,232 \\
\hline Femenino & $182(76,2 \%)$ & $57(23,8 \%)$ & \\
\hline Edad (años)* & $65(63-68)$ & $69(64-72)$ & $<0,001$ \\
\hline \multicolumn{4}{|l|}{ Nivel educativo } \\
\hline Hasta técnico & $278(73,2 \%)$ & $102(26,8 \%)$ & 0,094 \\
\hline Universitario & $18(90,0 \%)$ & $2(10,0 \%)$ & \\
\hline \multicolumn{4}{|c|}{ Diabetes Mellitus } \\
\hline No & $199(73,2 \%)$ & $73(26,8 \%)$ & 0,577 \\
\hline $\mathrm{Si}$ & $97(75,8 \%)$ & $31(24,2 \%)$ & \\
\hline \multicolumn{4}{|c|}{ Hipertensión arterial } \\
\hline No & $200(76,6 \%)$ & $61(23,4 \%)$ & 0,101 \\
\hline $\mathrm{Si}$ & $96(69,1 \%)$ & $43(30,9 \%)$ & \\
\hline \multicolumn{4}{|c|}{ Enfermedad renal crónica } \\
\hline No & $285(75,0 \%)$ & $95(25,0 \%)$ & 0,047 \\
\hline $\mathrm{Si}$ & $11(55,0 \%)$ & $9(45,0 \%)$ & \\
\hline \multicolumn{4}{|c|}{ Insuficiencia cardiaca } \\
\hline No & $235(80,2 \%)$ & $58(19,8 \%)$ & $<0,001$ \\
\hline $\mathrm{Si}$ & $61(57,0 \%)$ & $46(43,0 \%)$ & \\
\hline \multicolumn{4}{|c|}{ Lugar de residencia } \\
\hline Urbano & $93(69,4 \%)$ & $41(30,6 \%)$ & 0,137 \\
\hline Rural & $203(76,3 \%)$ & $63(23,7 \%)$ & \\
\hline
\end{tabular}

Los valores p se obtuvieron con la prueba de chi cuadrado y la suma de rangos (para la edad). *La edad fue tomada de forma cuantitativa y los valores que se muestran son las medianas (rangos intercuartílicos).

1,02-1,08; $\mathrm{p}<0,001)$, entre los que tenían diabetes (RPa: 1,23; IC95\%: 1,17-1,29; $\mathrm{p}<0,001$ ), hipertensión arterial (RPa: 1,47; IC95\%: 1,33-1,61; $<<0,001)$, enfermedad renal crónica (RPa: 2,21; IC95\%: 2,01-2,43; $\mathrm{p}<0,001)$ e insuficiencia cardiaca (RPa: 2,25; IC95\%: 1,92-2,64; $p<0,001)$; en cambio, los que tenían grado universitario tuvieron menos deterioro cognitivo (RPa: 0,89; IC95\%: $0,22-0,65 ; \mathrm{p}<0,001)$; ajustados por el sexo y el lugar de residencia. Tabla 3

\section{DISCUSIÓN}

Uno de cada cuatro adultos mayores tuvo deterioro cognitivo moderado y menos del $1 \%$ de forma grave, a diferencia de un estudio realizado en Hospitales de España, donde solamente el 39\% presentó deterioro cognitivo, además de que, los pacientes estudiados fueron de mayor edad (edad media de 77,9 $\pm 9,8$ años) (14).

A mayor edad hubo mayor deterioro cog- 
Tabla 3. Análisis bivariado y multivariado de los factores asociados al deterioro cognitivo en adultos mayores de Cochabamba-Bolivia.

\begin{tabular}{lcc}
\hline Variables & Análisis bivariado & Análisis multivariado \\
\hline Sexo femenino & $0,82(0,80-0,83)<0,001$ & $0,90(0,72-1,14) 0,383$ \\
Edad (años) & $1,07(1,02-1,12) 0,004$ & $1,05(1,02-1,08)<0,001$ \\
Universitarios & $0,37(0,18-0,76) 0,006$ & $0,38(0,22-0,65)<0,001$ \\
Diabetes mellitus & $0,90(0,82-0,99) 0,026$ & $1,23(1,17-1,29)<0,001$ \\
Hipertensión arterial & $1,32(0,86-2,03) 0,197$ & $1,47(1,33-1,61)<0,001$ \\
Enfermedad renal crónica & $1,80(1,39-2,33)<0,001$ & $2,21(2,01-2,43)<0,001$ \\
Insuficiencia cardiaca & $2,17(1,34-3,52) 0,002$ & $2,25(1,92-2,64)<0,001$ \\
\hline
\end{tabular}

Las razones de prevalencia (intervalos de confianza al 95\%) y valores p fueron obtenidos con los modelos lineales generalizados (familia Poisson, función de enlace log, modelos robustos y ajustados por el lugar de residencia). La variable edad es de tipo cuantitativa.

nitivo moderado o severo, al igual que lo reportado en el estudio realizado en Chile, donde los principales factores asociados a sospecha de deterioro cognitivo fueron la edad, el bajo nivel de escolaridad y el sexo masculino $(15,16)$; lo que implica mayores riesgos en el manejo hospitalario $(17,18)$.

Los que tenían estudios universitarios tuvieron menor deterioro cognitivo moderado o severo, que concuerda con los estudios realizados en Italia, donde mostraron la tasas de mortalidad más altas para educación primaria o inferior (19).

Los que tenían una de las cuatro comorbilidades evaluadas tuvieron un mayor deterioro cognitivo moderado o severo. En Chile encontraron que la asociación es mayor en individuos diabéticos y/o con historia familiar de diabetes mellitus (20), esto es importante como se demuestra en el estudio de seguimiento que se realizó en Japón, donde la tasa de mortalidad aumentó con el número adicional de factores de riesgo, siendo el deterioro cognitivo un predictor de mortalidad (21).

Es importante resaltar que los adultos mayores tienen varias comorbilidades, como se observa en el estudio nacional realizado en Estados Unidos en la población de 62 a 90 años de edad, donde el numero promedio fue de 2,7 patologías (22).

No se ha estudiado lo suficiente sobre el deterioro cognitivo en lugares con mediana altura geográfica, en China a una altitud de 2275 metros sobre el nivel del mar, el 24\% de sus pacientes estudiados tenían deterioro cognitivo (23) a diferencia de nuestro estudio que en una mayor altitud (2500-3900 msnm) y pacientes con menor edad, se encontró que el $73,5 \%$ presento algún grado de deterioro cognitivo.

El estudio tuvo la limitación del sesgo de selección, ya que, el muestreo no permite extrapolar los datos a toda la población adulta mayor de Cochabamba, ni a otras de alturas similares, sin embargo, por la importante muestra y las asociaciones encontradas se puede tomar este reporte como un importante precedente, siendo labor de las instituciones de salud la de liderar estudios con una mayor población y de sedes. Sin embargo, debido a la buena potencia del cruce de las variables si se pueden usar los resultados analíticos, por lo que, esto puede ayudar a futuras investigaciones e instituciones de la altura geográfica que busquen evaluar los factores asociados al deterioro cognitivo.

Por todo lo mencionado se concluye que, 
el $25 \%$ de adultos mayores que viven en una altura geográfica intermedia tienen un deterioro cognitivo moderado, el $0,5 \%$ tiene un deterioro cognitivo severo. A mayor edad hubo mayor deterioro cognitivo moderado o severo, así como, los que tenían una comorbilidad. Los que tenían estudios universitarios tuvieron menor deterioro cognitivo moderado o severo.

\begin{abstract}
Resumen
Introducción: El deterioro cognitivo es una patología importante entre los ancianos, pero pocos estudios evalúan a poblaciones en una altura geográfica importante. Objetivos: Caracterizar y encontrar los factores asociados al deterioro cognitivo en poblaciones urbanas y rurales de la mediana altura geográfica. Metodología: Estudio transversal analítico, se evaluó el deterioro cognitivo con el Test de Pfeiffer (Alpha de Cronbach: 0,69), sus resultados se combinaron versus tres variables socioeducativas y cuatro comorbilidades; se obtuvo estadísticos analíticos. Resultados: De los 400 adultos mayores, el $26,5 \%$ (106) no tuvo deterioro cognitivo, el $47,5 \%$ (190) tuvo un deterioro leve, el $25,5 \%$ (102) moderado y el 0,5\% (2) severo; el $60 \%$ fueron mujeres, la mediana de edades fue de 66 años (rango intercuartílico: 63-69 años), el 67\% vivían en la zona rural. En el análisis multivariado se encontró que, hubo más deterioro cognitivo moderado/severo a mayor edad (RPa: 1,05; IC95\%: 1,02-1,08; $p<0,001$ ), entre los que tenían diabetes (RPa: 1,23; IC95\%: 1,17-1,29; $p<0,001)$, hipertensión arterial (RPa: 1,47; IC95\%: 1,33-1,61; p<0,001), enfermedad renal crónica (RPa: 2,21; IC95\%: 2,01-2,43; p<0,001) e insuficiencia cardiaca (RPa: 2, 25; IC95\%: 1,922,64; $p<0,001)$; en cambio, los que tenían grado universitario tuvieron menos deterioro cognitivo (RPa: 0,89; IC95\%: 0,22-0,65; $p<0,001$ ); ajustados por el sexo $y$ el lugar de residencia. Conclusiones: Se encontró importantes asociaciones del deterioro cognitivo en una población adulta mayor que residía entre los 2500-3900 msnm, estos resultados son importantes por no ser una población muy estudiada.
\end{abstract}

Palabras clave: Disfunción Cognitiva, Altitud, Ancianos.

\section{REFERENCIAS BIBLIOGRÁFICAS}

1. ParkarSR. Elderly MentalHealth: Needs. Mens Sana Monogr. 2015;13(1):91-9.

2. Rebagliati Tirado C. Caracterización clínica, epidemiológica y social de la población geriátrica hospitalizada en el Centro Geriátrico Naval. Enero 2010 - noviembre 2011. [Internet] [Tesis de
Grado]. [Perú]: Universidad Cientifica del Sur; 2012 [citado 25 de mayo de 2020]. Disponible en: https://repositorio. cientifica.edu.pe/handle/UCS/175

3. Chandía Reyes VA, Luengo Martínez C, Chandía Reyes VA, Luengo Martínez C. Relación entre hipertensión arterial y diabetes mellitus tipo 2 con deterioro cognitivo en adultos mayores. Geroko- 
mos. 2019;30(4):172-5.

4. Hota SK, Sharma VK, Hota K, Das S, Dhar P, Mahapatra BB, et al. Multi-domain cognitive screening test for neuropsychological assessment for cognitive decline in acclimatized lowlanders staying at high altitude. Indian $\mathrm{J}$ Med Res. 2012;136(3):411-20.

5. Evans IEM, Llewellyn DJ, Matthews FE, Woods RT, Brayne C, Clare L. Social isolation, cognitive reserve, and cognition in healthy older people. PLoS One. 2018;13(8):e0201008.

6. Malinauskiene V, Leisyte P, Romualdas M, Kirtiklyte K. Associations between self-rated health and psychosocial conditions, lifestyle factors and health resources among hospital nurses in $\mathrm{Li}$ thuania. Journal of advanced nursing. 2011;67(11):2383-2393.

7. Véliz M. Efectos del Envejecimiento en los procesos de comprensión y producción de Lenguaje. Paideia. 2020;1(54):11-32.

8. Blasco CB, Ribes JV. Neurofisiología y envejecimiento. Concepto y bases fisiopatológicas del deterioro cognitivo. Revista Española de Geriatría y Gerontología. 2016;51(1):3-6.

9. 9. Instituto Nacional de Estadistica de Bolivia. Has buscado demografia [Internet]. INE. [citado 25 de mayo de 2020]. Disponible en: https://www.ine.gob.bo/ index.php/search/demografia/

10. De la Iglesia JM, Dueñas Herrero R, Onís Vilches MC, Aguado Taberné C, Albert Colomer C, Luque Luque R. Adaptación y validación al castellano del cuestionario de Pfeiffer (SPMSQ) para detectar la existencia de deterioro cognitivo en personas mayores de 65 años. Medicina Clínica. 2001;117(4):129-34.

11. Pfeiffer E. A Short Portable Mental Status Questionnaire for the Assessment of Organic Brain Deficit in Elderly Patients $\dagger$. Journal of the American Geria- trics Society. 1975;23(10):433-41.

12. Fillenbaum G, Heyman A, Williams K, Prosnitz B, Burchett B. Sensitivity and specificity of standardized screens of cognitive impairment and dementia among elderly black and white community residents. Journal of Clinical Epidemiology. 1990;43(7):651-60.

13. Moreno-Gaviño L, Ruiz-Cantero A, Bernabeu-Wittel M, Tejera-Concepción A, Romero-Jiménez M, Soria MÁ, et al. Impact of Cognitive Impairment in a Multicentric Cohort of Polypathological Patients. International Journal of Gerontology. 2012;6(2):84-9.

14. Bernabeu-Wittel M, Nieto Martín D, Moreno-Gaviño L, Ollero-Baturone M. Valor diagnóstico de un cuestionario de Pfeiffer simplificado en pacientes pluripatológicos. Rev Clin Esp. 2017;217(6):320-4.

15. Martínez-Sanguinetti MA, Leiva AM, Petermann-Rocha F, Troncoso-Pantoja C, Villagrán M, Lanuza-Rilling F, et al. Factores asociados al deterioro cognitivo en personas mayores en Chile. Revista médica de Chile. 2019;147(8):1013-23.

16. Cancino M, Rehbein-Felmer L, Ortiz MS, Cancino M, Rehbein-Felmer L, Ortiz MS. Funcionamiento cognitivo en adultos mayores: rol de la reserva cognitiva, apoyo social y depresión. Revista médica de Chile. 2018;146(3):315-22.

17. Linares-Nolasco F, Mille-Loera JE, Sosa-Jaime NA. Anestesia general y alteraciones cognitivas en el paciente geriátrico. Rev Mex Anest. 2019;42(S1):40-1.

18. Shami A, Brennan M, Marie PS, Lindenauer PK, Stefan MS. The association of cognitive impairment as screened by the Mini-Cog with long term post-hospitalization outcomes. Arch Gerontol Geriatr. 2019;85(1):103916.

19. Zwieten A van, Wong G, Ruospo M, Palmer SC, Teixeira-Pinto A, Barulli MR, et al. Associations of Cognitive Func- 
tion and Education Level With All-Cause Mortality in Adults on Hemodialysis: Findings From the COGNITIVE-HD Study. American Journal of Kidney Diseases. 2019;74(4):452-62.

20. Petermann F, Troncoso-Pantoja C, Martínez MA, Leiva AM, Ramírez-Campillo R, Poblete-Valderrama $F$, et al. [Risk of cognitive impairment among older people with diabetes or family history of the disease]. Rev Med Chil. 2018;146(8):872-81.

21. Igari R, Davy P, Sato H, Takahashi Y, Iseki $\mathrm{C}$, Kato $\mathrm{H}$, et al. Cognitive impairment, brain ischemia and shorter telomeres are predictors of mortality in the Japanese elderly: A 13-year prospective community-based study. J Neurol Sci. 2019;397(1):129-34.
22. Kotwal AA, Kim J, Waite L, Dale W. Social Function and Cognitive Status: Results from a US Nationally Representative Survey of Older Adults. J Gen Intern Med. 2016;31(8):854-62.

23. Mei L, Wu S, Wang D, Li H, Zhang H, Wang M. Epidemiology of Dementia in Elderly Chronic Obstructive Pulmonary Disease Patients Living in China's Northwestern High-Elevation Area. Med Sci Monit. 2018;24(1):7742-9.
Correspondencia:

Christian R. Mejia

Av. Las Palmeras 5713, Los Olivos, Lima, Perú. CP: 15304

(+511) 997643516

christian.mejia.md@gmail.com 